CORRECTION

Check for updates

\title{
Correction to: Eosinophilic vacuolated tumor (EVT) of kidney demonstrates sporadic TSC/MTOR mutations: next-generation sequencing multi-institutional study of 19 cases
}

Mihaela Farcaș, Zoran Gatalica, Kiril Trpkov (D), Jeffrey Swensen, Ming Zhou, Reza Alaghehbandan, Sean R. Williamson (D), Cristina Magi-Galluzzi (D), Anthony J. Gill, Maria Tretiakova (D), Jose I. Lopez (D), Delia Perez Montiel, Maris Sperga, Eva Comperat, Fadi Brimo, Asli Yilmaz, Farshid Siadat, Ankur Sangoi, Yuan Gao, Nikola Ptákova (D), Levente Kuthi, Kristyna Pivovarcikova, Joanna Rogala, Abbas Agaimy (D), Arndt Hartmann, Cristoph Fraune, Boris Rychly, Pavel Hurnik, Dušan Durcansky, Michael Bonnert (D), Georgios Gakis, Michal Michal, Milan Hora and Ondrej Hes (D)

(c) The Author(s), under exclusive licence to United States \& Canadian Academy of Pathology 2021

Modern Pathology (2021) 34:2243; https://doi.org/10.1038/s41379-021-00941-4

Correction to: Modern Pathology; https://doi.org/10.1038/s41379021-00923-6, published online 14 September 2021

The original version of this article unfortunately containted a mistake in the author names. The authors' names were presented with the surnames in the original paper. The following list is in the correct style for Modern Pathology. Mihaela Farcaş, Zoran Gatalica, Kiril Trpkov, Jeffrey Swensen, Ming Zhou, Reza Alaghehbandan,
Sean R. Williamson, Cristina Magi-Galluzzi, Anthony J. Gill, Maria Tretiakova, Jose I. Lopez, Delia Perez Montiel, Maris Sperga, Eva Comperat, Fadi Brimo, Asli Yilmaz, Farshid Siadat, Ankur Sangoi, Yuan Gao, Nikola Ptákova, Levente Kuthi, Kristyna Pivovarcikova, Joanna Rogala, Abbas Agaimy, Arndt Hartmann, Cristoph Fraune, Boris Rychly, Pavel Hurnik, Dušan Durcansky, Michael Bonnert, Georgios Gakis, Michal Michal, Milan Hora, and Ondrej Hes. The original article has been corrected. 